\title{
Megachiropteran Bats (Pteropus) Utilize Human Referential Stimuli To Locate Hidden Food
}

\begin{abstract}
Nathaniel J. Hall, Monique A. R. Udell, Nicole R. Dorey, and Allyson L. Walsh University of Florida

Spontaneous point-following behavior has been considered an indicator of advanced social cognition unique to humans. Recently, it has been suggested that a close evolutionary relationship with humans could result in similar social skills in domesticated species. An alternative view is that the mechanism is not genetic domestication alone but instead a combination of phylogenetic and ontogenetic variables. Here we test the necessity of phylogenetic domestication by investigating the point-following behavior of a captive population of nondomesticated megachiropteran bats (Pteropus pumilus, Pteropus rodricensis, Pteropus conspicillatus, Pteropus vampyrus). Three of five subjects were highly successful in following an unfamiliar human's point to a target location, providing the first empirical evidence of cross-species social referencing in bats. The three successful bats were all born in captivity and socialized to humans early in life, whereas unsuccessful bats were wild-born individuals. This study provides evidence that referential point following is not restricted to domesticated animals and indicates that early experience may be important. Megachiropteran bats may prove to be a useful model for studying social behaviors.
\end{abstract}

\author{
Clive D. L. Wynne \\ University of Florida and Kyung Hee University
}

Keywords: megachiropteran bats, social cognition, human gestures, social referencing

Joint attention and point following are considered important markers of sociocognitive development in human infants and are often used as indicators for theory of mind capabilities in preverbal human children (Carpenter, Nagell \& Tomasello, 1998; Charman et al., 2000). Recently, comparative research on spontaneous point-following behavior in an object-choice task has been used to support the claim that domestic dogs, and possibly domesticated species in general, have evolved human-like social skills (Hare \& Tomasello, 2005). This domestication hypothesis proposes that selection pressures present during domestication led to the development of heritable human-like social skills that increased genetic fitness (Hare, Brown, Williamson, \& Tomasello, 2002; Miklósi et al., 2003). Thus, dogs, and domesticated species in general, have evolved a specialized capacity to read human social and communicative behavior (Hare \& Tomasello, 2005).

Support for the domestication hypothesis comes from evidence demonstrating that some domesticated species (goats, Capra hirucs; horses, Equus caballus; cats, Felis catus) show proficiency in following a human point to a target without explicit training (for

Nathaniel J. Hall, Monique A. R. Udell, and Nicole R. Dorey, Department of Psychology, University of Florida; Allyson L. Walsh, Department of Wildlife Ecology and Conservation, University of Florida; Clive D. L. Wynne, Department of Psychology, University of Florida, and Kyung Hee University, Seoul, South Korea.

We are grateful for the help and support provided by The Lubee Bat Conservancy, Gainesville, Florida, and thank them for the use of their animals and facilities; specifically, we thank Brian Pope and Tasha King, who contributed greatly to this project.

Correspondence concerning this article should be addressed to Monique A. R. Udell, University of Florida, Department of Psychology, P.O. Box 112250, Gainesville, FL 32611. E-mail: mudell@ufl.edu goats, see Kaminski, Riedel, Call, \& Tomasello, 2005; for horses, see Maros, Gácsi, \& Miklósi, 2008; McKinley \& Sambrook, 2000; for cats, see Miklósi, Pongrácz, Lakatos, Topál, \& Csányi, 2005). However, to determine if domestication is necessary for the development of responsiveness to human social and communicative gestures, adequate comparisons to the social behavior of nondomesticated species must also be made.

Most of the available literature comparing domesticated and nondomesticated species' point-following behavior has focused on canids, specifically pet dogs (Canis lupus familiaris) and wolves (C. l. lupus; Agnetta, Hare, \& Tomasello, 2000; Gácsi et al., 2009; Hare et al., 2002; Miklósi et al., 2003; Udell, Dorey, \& Wynne, 2008; Virányi et al., 2008). Some researchers have found that wolves do indeed spontaneously follow human pointing gestures (Gácsi et al., 2009; Udell et al., 2008), while others report that untrained wolves perform at chance levels (Hare et al., 2002; Virányi et al., 2008). In a related line of investigation, Hare et al. (2005) reported that silver foxes artificially bred over many generations for tame behavior (Trut, 1999) were spontaneously more sensitive to human pointing gestures than wild-type foxes. However, the wild-type foxes in this study nonetheless followed the experimenter's point more often than would be expected by chance, suggesting that domestication may have influenced performance but did not predict absolute success or failure on the task. While there is strong evidence for the interplaying roles of phylogeny and ontogeny in the development of dogs' social skills (Udell \& Wynne, 2010), the precise role of domestication is still an ongoing debate within the canid literature.

Other nondomesticated species tested for sensitivity to human gestures include dolphins (Tursiops truncates) and fur seals (Arctocephalus pusillus; Pack \& Herman, 2004; Scheumann \& Call, 2004; Tschudin, Call, Dunbar, Harris, \& van der Elst, 2001). 
While some subjects in these studies utilized human points at above chance levels, their status as animals trained for public display or other experiments has sometimes made direct comparisons with untrained domesticated animals difficult (Miklósi \& Soproni, 2006). More recently, an experiment conducted with a species known to engage in cooperative social behavior in the wild, jackdaws (Corvus monedula), demonstrated that hand-raised individuals would spontaneously follow a point issued by their familiar caretaker (Von Bayern \& Emery, 2009). The findings of this study suggest that giving a species that engages in cooperative social behaviors with conspecifics early experiences with humans may be important for spontaneous point-following behavior. The degree to which point-following behaviors may extend to unfamiliar experimenters is unknown.

Both megachiropteran and microchiropteran bats are considered generally social (for a review, see Kerth, 2008). Various bat species have been recorded engaging in conspecific grooming, transfer of information, reciprocal food sharing, and mutual warming (Kerth, 2008; McCracken \& Bradbury, 1981; Safi \& Kerth, 2007; Wilkinson, 1984, 1986, 1992). Of the species in this study, Pteropus rodricensis have been recorded aiding conspecifics in parturition (Kunz \& Allgaier, 1994). In addition, Pteropus vampyrus are considered a gregarious species (Kunz \& Jones, 2000). Unfortunately, detailed information on many bat species' social organization is sparse compared to what is known of other social species (Kerth, 2008). Furthermore, megachiropterans have vision adequate to participate in traditional object choice tasks (Müller, Goodman, \& Piechl, 2007).

In this study, we investigated the performance of five captive bats, belonging to the suborder Megachiroptera, in an object choice task that required them to spontaneously utilize the point of an unfamiliar human to locate a hidden target.

\section{Method}

\section{Subjects/Setting}

Five Megachiroptera from four different species currently residing at a bat conservancy were selected for participation in this study. The bats were kept in outdoor, enclosed octagonal pens (side length of $4.52 \mathrm{~m}$ ) with other bats of varying species. While a familiar keeper acted as an assistant, calling or returning the subject back to the starting point between trials, the experimenter who issued the pointing gesture during experimental trials was previously unfamiliar to the bat subjects.

Three of the five bats were captive-born (Pteropus pumilus, Pteropus rodricensis, Pteropus conspicillatus), while two bats were wild-caught (Pteropus pumilus, Pteropus vampyrus). The captive-born bats had varying histories (mother reared or hand reared on or off the premises of the conservancy) but are distinct from wild-caught bats in that they were born in captivity, provided regular interactions with humans from birth, and reached adulthood in captivity. Wild-caught bats matured to adulthood before being captured and brought to the conservancy but had spent about 16 years in captivity prior to testing. The captive-born bats were also mature adults, at 8, 10, and 11 years of age, and had spent their entire lives in captivity. Thus, the major difference between the groups was that captive-born bats had early experiences with humans handling or entering their enclosure, while wild-caught bats did not. Once at the conservancy, wild-caught and captiveborn bats cohabitated and had similar experiences with humans and feeding.

\section{Materials}

The bats were tested individually in vacant triangular outdoor pens $(6.2 \mathrm{~m} \times 4.52 \mathrm{~m} \times 4.52 \mathrm{~m})$. Two opaque plastic containers served as the response objects and were fixed $1.6 \mathrm{~m}$ apart at an appropriate height for the individual bat to reach (see Figure 1). Each response object held a 250-ml Mason jar that was out of sight of the bat (see Figure 2). Both Mason jars contained $1 \mathrm{ml}$ of Kern's Nectar ${ }^{\mathrm{TM}}$ (Kern's Beverages, Santa Ana, CA). The lids normally used to seal Mason jars were removed, but the rims used to affix the lid to the jars were retained for the purposes of this study.

The rims were placed on both Mason jars, however, one jar's rim was fitted with a fiberglass screen (sham rim) while the other jar's rim was not manipulated (free rim; Figure 2A). The screen on the sham rim permitted airflow from both jars, and hence equalized smell from the jars, while preventing the bat access to the nectar in the sham jar. The other rim provided free access to the nectar. As demonstrated previously, hiding equal amounts of nectar in each box was necessary, because some species of Megachiroptera cannot only determine the location of food by odor alone in a two choice experiment but can even discriminate whether the fruit is ripe (Luft, Curio, \& Tacud, 2003). One bat in our study, Easter, was also initially suspected of using additional scent cues that she may have left on the rim of the free lid when allowed to access the free food during her first set of control trials. This potential confound was resolved by cleaning both jars between each trial, both control and experimental, after which her performance on control trials dropped to chance, while her performance on experimental trials remained significantly above chance.

\section{Experimental Testing}

Prior to participation in the study, each bat was required to readily approach and take food from the experimenter and experimental apparatus when it was freely available. This was done on the day of testing by the experimenter holding up a container of

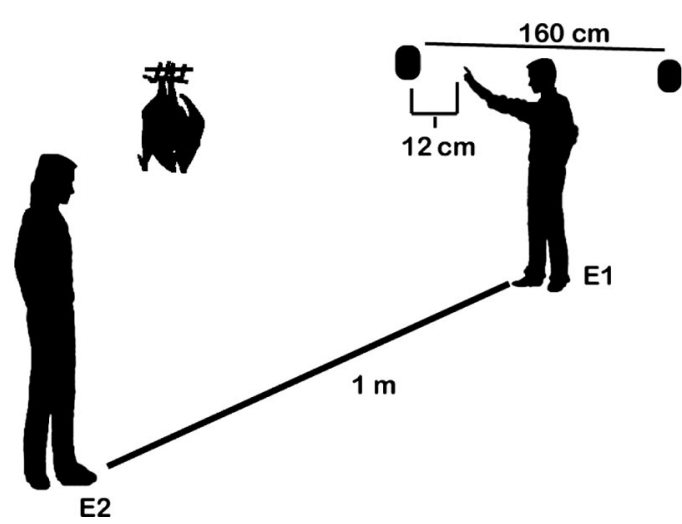

Figure 1. Testing layout. Experimenter 1 (E1) pointed to the target object and continued to point until the bat made a choice. A familiar caretaker, Experimenter 2 (E2), served as the call-back experimenter. E2 retrieved the bat and placed the bat at the correct starting place before every trial began. 


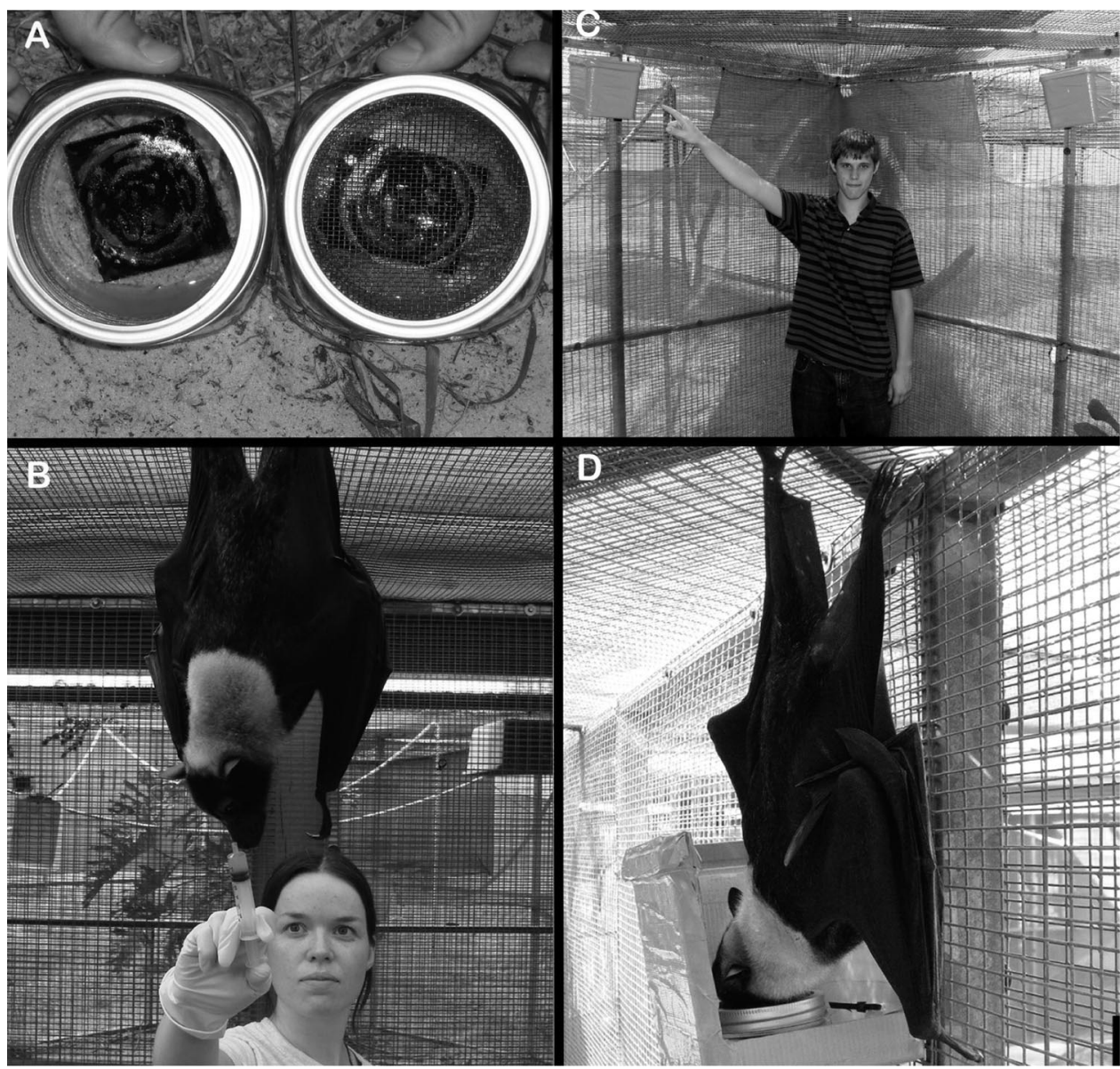

Figure 2. Experimental materials and design. (A) The jar on the left has an open lid, making the nectar inside accessible; this jar was placed in the target container. The right jar has a wire mesh lid, making the nectar inaccessible; this jar was located in the incorrect testing container. (B) A subject being recalled to the start by the assistant. Bats traveled between the assistant and experimenter by pulling themselves across the roofing. (C) An unfamiliar experimenter is making a dynamic proximal point toward the target container. (D) The subject has made a correct response, entered the target container, and is obtaining nectar.

nectar next to the bat. If the bat approached the container, the container was placed inside one of the experimental boxes. This procedure was repeated until the bat consumed food from each experimental box twice. The procedure ensured that the bat subjects were food motivated and were not fearful or distracted within the testing environment. Four bats never approached the experimenter or the experimental apparatus despite being given hours to habituate to the apparatus. These four bats never began testing.

Each experimental trial began with the assistant bringing the bat to the starting location $1 \mathrm{~m}$ back from the experimental boxes (Figure 2B). Once the bat was in position, the experimenter raised his ipsilateral arm to chest height and directed his hand toward the bat. The point started this way to allow for a range of motion of the arm and to gain the bat's attention. The experimenter called the bat's name and then moved his arm laterally in the direction of the correct box. Movement stopped once the arm was pointing to the correct box and the finger was approximately $12 \mathrm{~cm}$ from the box (Figure 2C). The gesture, a dynamic proximal point as defined by Miklósi \& Soproni (2006), did not move further until the bat made a choice or the trial timed out after $2 \mathrm{~min}$. The point is considered dynamic because the subject is able to see the movement of the point, and the arm remains in the gesturing position throughout the trial. While gesturing, the experimenter looked straight ahead and avoided eye contact.

Trials lasted $2 \mathrm{~min}$ to give the bats sufficient time to move from the start location to the target container by pulling themselves across the enclosure roof (the bats' locomotor method of choice) in a climbing motion. None of the bats flew in the experimental pen during testing. An observer outside the enclosure recorded the bats' choice for each trial as it occurred. The observer recorded a choice when the bat physically touched one of the response objects (Figure 2D), which were located $1.6 \mathrm{~m}$ apart. In every trial where a bat made a choice, the bat subsequently entered the response object to obtain accessible or inaccessible nectar. Thus, the bats' choice between response objects was unambiguous. If a bat made a correct choice, but had difficulties in consuming the nectar, the experimenter assisted the bat by lifting the jar to increase accessibility. If the bat made an incorrect choice, the assistant called the 
bat back to the start position without it receiving nectar. If the bat moved toward the testing apparatus but had not made a choice within two minutes, the assistant called the bat back to start the next trial and a "no choice" was recorded. No choice responses were analyzed as incorrect responses. Only one "no choice" response occurred during experimental trials (Easter, Session 1).

After each trial, the experimenter removed both jars from the response objects. If the bat had made a correct choice on the previous trial, and subsequently consumed the nectar from the jar in the target container, the experimenter removed both lids from the jars and refilled the emptied jar with $1 \mathrm{ml}$ of nectar. If the bat had made an incorrect choice on the previous trial (and therefore did not consume the nectar), the experimenter removed the jars and the lids but did not add any more nectar to the jars. Out of the view of the bat, the experimenter simultaneously replaced both lids and placed the lid allowing access to the nectar on the appropriate jar that was then put into the target container. Both jars were then placed in their respective boxes.

The target container for each trial was determined pseudorandomly, with the stipulation that no side could be used more than twice in a row or for more than $50 \%$ of the trials. Each subject received a total of 20 experimental trials, divided into two sessions of 10 trials each. If a bat satiated with nectar, experimental trials for that day were suspended. Satiation was determined when the bats failed to take food offered by the assistant (a familiar caretaker). Experimental trials were resumed on another day. Four of the five bats participating in experimental trials completed testing, while one bat, Arthur, refused to participate after the first six trials.

\section{Control Trials}

Three to seven control trials followed every 10 experimental trials. Control trials were identical to experimental trials, except that no pointing cue was administered.

\section{Statistical Analysis}

A one-sample $t$-test was used to determine if the group performance of the bats was better than would be expected by chance within each session. A paired $t$-test was used to determine if performance differed between session one and session two. Both tests were two-tailed and had an alpha level of .05. Binomial tests were used to assess the individual performance of each bat for each session, with success criterion set at $8 / 10$ trials correct or better within an individual session $(p \leq .05)$. Analyses were conducted using Excel and SPSS.

\section{Results}

As a group, the bats were successful in following a human point in each testing session (one sample $t$-test, Session $1, \mathrm{t}_{3}=4.37, p=$ .022 ; Session 2, $\left.\mathrm{t}_{3}=7.35, p=.005\right)$. At the individual level, all three captive-born bats, Grace, Kuri, and Easter, were successful in reliably following a human point to the target in each session (Session 1 and Session 2, binomial test, $p<.05$, see Figure 3) and pooled across both sessions (Grace, 16 out of 20, $p \leq .01$; Kuri, 18 out of $20, p<.01$; Easter, 16 out of $20, p \leq .01$ ). Neither wild-caught bat performed significantly above chance on the task. Arthur, one of the wild-caught bats, began testing but only com-

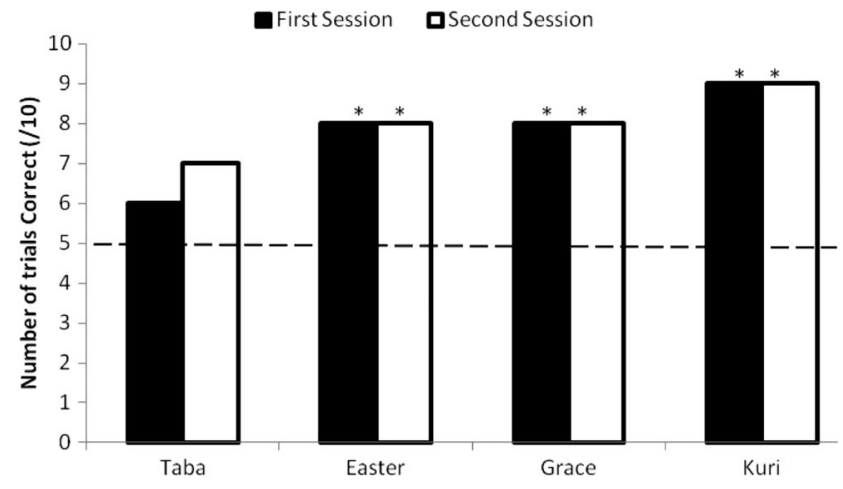

Figure 3. Individual performance in each testing session. The number of successful trials, out of 10 for each session, for each bat that completed testing is displayed. Three captive-born bats (Easter, Grace, and Kuri) and one wild-caught bat (Taba) are shown. The second wild-caught bat (Arthur) did not complete testing (completed only six trials, two of which were correct choices) and is not shown in this figure. * indicates performance significantly above chance in that session (binomial test, $p \leq .05$ ). The dashed line indicates chance responding on the task.

pleted six experimental trials before refusing to participate, even after multiple breaks and revisits on other days. Out of those six completed trials, he chose correctly only twice $(33 \%$ correct). Taba, the other wild-caught bat, readily approached a container in search of the accessible food on every trial; however, her performance never reached statistical significance (Session 1 and Session 2, binomial test, $p>.05$ ).

We also analyzed first-trial performance for each bat, and compared performance in the first session to that in the second session of testing to assess whether performance was more likely a product of a bat's capacity before testing or learning within the course of the experiment. There was no difference in performance between the first and second sessions of testing (paired $t$-test, $\mathrm{t}_{3}=1.00, p=$ .39). A bat's first response on the first trial was not a good predictor of subsequent performance. The three successful bats (Easter, Grace, and Kuri) made a correct choice on the first trial of testing; so did one of the unsuccessful bats, Taba. Arthur was the only bat to make an incorrect response on the first trial.

Control trials were conducted during and after testing in which the bat was allowed to choose a container in the absence of a human point. Bats performed at chance levels on control trials, both on average and at the individual level (mean performance on control trials for all subjects, $49 \%$ correct, binomial test, $p=.50$ ), indicating that they were not locating the accessible food based on smell or unintentional cueing by the experimenter. As mentioned above, Easter was initially suspected of using smell cues to locate the target container on control trials. This was resolved by cleaning the lids and jars between trials. Prior to cleaning the lids, she scored 8 correct out of 10 trials; after the introduction of the cleaning lid method, performance on control trials dropped to chance, 6 out of 14 (43\% correct, binomial test, $p=.77$ ). Performance on experimental trials remained above chance despite cleaning between trials ( $80 \%$ correct, binomial test, $p \leq .05$ ). The control trials of the other bats did not indicate the use of any unintentional cues (Taba, Session 1 and Session 2, 50\% correct on controls; Grace, Session 1 and Session 2, 25\% correct; Kuri, 
Session 1 and Session 2, 55\% correct; Arthur did not complete control trials).

\section{Discussion}

These results demonstrate that several species of experimentally naïve bats are capable of spontaneously utilizing human points to find the location of concealed food in an object-choice task. This suggests that domestication is not necessary for the responsiveness to the human gesture tested. Instead, the proclivity of bats toward conspecific social interactions may be important in understanding their ability to engage in heterospecific interactions such as following human points. Nonetheless, responsiveness to the actions of heterospecifics does not appear to be automatic. Human socialization and regular interaction with humans from an early age emerged as an important predictor of an individual bat's performance. All three captive-born individuals that participated in the experiment followed the experimenter's point to the target location successfully. Both wild-born bats were unsuccessful on the task despite having spent 16 years of their adulthood in captivity.

Early and intensive exposure to humans has been linked to the success of other nondomesticated species on human-guided tasks (Udell et al., 2008; Von Bayern \& Emery, 2009). Taken together with this study, this may suggest that there is an important sensitive period for socialization that can help predict success on human-guided tasks. However, an additionally important factor in this study was that the successful bat subjects were able to follow the point of an unfamiliar experimenter, demonstrating the ability to generalize their response to referential stimuli provided by humans in general.

While in previous reports, chimpanzees have been reported to perform at lower levels than both human children and domestic dogs on human guided tasks (Hare et al., 2002; Bräuer, Kaminski, Riedel, Call, \& Tomasello, 2006), this might have been due, at least in part, to significant differences in the quantity and quality of daily exposure to humans. Differences between enculturated and wild-born chimpanzees indicate environmental factors contribute to the ontogeny of primate social cognition (Tomasello \& Call, 2004). Tomasello and Call (2004) hypothesized that growing up in the presence of humans changed the way individuals attended and reacted to human actions, and enculturation acted as a catalyst for further development of an already-present sociocognitive capacity (Tomasello \& Call, 2004).

The individual socialization experiences of the bats in this study differ only at birth until one to two years of age. Captive-born individuals received at least some close human interaction from birth, where wild-caught bats did not. Experiences as adults in captivity were nearly identical for all subjects. The hypothesized role of early socialization in the development of responsiveness to human gestures is consistent with the differences found between our captive-born and wild-caught bats thus far.

The success of species generally considered social may indicate an important phylogenetic component to point-following behavior in object choice tasks. Species that regularly interact with conspecifics may be more apt at cooperative interactions with heterospecifics. Thus a two-pronged strategy may be needed to further identify species and individuals likely to display sensitivity to human gestures: (a) recognizing species characteristics that suggest a phylogenetic capacity, including evidence of conspecific social interactions of the species, while (b) also taking into account ontogenic factors.

With the current sample size, accurate first-trial responding was difficult to interpret conclusively; however, larger sample sizes might be more conducive to a first-trial analysis and may be able to provide information about spontaneous responding in future studies. In addition, further research is needed to determine whether the bats' performance should be explained as a learned association between human hands and the presentation of food, a form of local enhancement, stimulus enhancement, or as requiring an understanding of reference or intentionality. More point types should also be used in future studies to ascertain whether success with proximal pointing would extend to more distal points. With the current data, we propose that the social proclivity of bats and early exposure to humans during ontogeny both likely contributed to the development of increased responsiveness to humans.

Bats could serve as an important nondomesticated animal model for investigating the origins of human-like social cognition. More generally, bats are rich but underrepresented subjects in animal cognition research and ought to be better represented in future studies.

\section{References}

Agnetta, B., Hare, B., \& Tomasello, M. (2000). Cues to food location that dogs (Canis familiaris) of different ages do and do not use. Animal Cognition, 3, 107-112.

Bräuer, J., Kaminski, J., Riedel, J., Call, J., \& Tomasello, M. (2006). Making inferences about the location of hidden food: Social dog, causal ape. Journal of Comparative Psychology, 120, 38-47.

Carpenter, M., Nagell, K., \& Tomasello, M. (1998). Social cognition, joint attention, and communicative competence from 9 to 15 months of age. Monographs of the Society for Research in Child Development, 6, $1-174$.

Charman, T., Baron-Cohen, S., Swettenham, J., Baird, G., Cox, A., \& Drew, A. (2000). Testing joint attention, imitation, and play as infancy precursors to language and theory of mind. Cognitive Development, 15, 481-498.

Gácsi, M., Györi, B., Virányi, Z., Kubinyi, E., Range, F., Belényi, B., \& Miklósi, Á. (2009). Explaining dog wolf differences in utilizing human pointing gestures: Selection for synergistic shifts in the development of some social skills. Plos One, 4, e6584, doi:10.1371/journal.pone .0006584

Hare, B., Brown, M., Williamson, C., \& Tomasello. M. (2002, November 22). The domestication of social cognition in dogs. Science, 298, 1634 1636.

Hare, B., Plyusnina, I., Ignacio, N., Schepina, O., Stepika, A., Wrangham, R., \& Trut, L. (2005). Social cognitive evolution in captive foxes is a correlated by-product of experimental domestication. Current Biology, $15,226-230$.

Hare, B., \& Tomasello, M. (2005). Human like social skills in dogs? Trends in Cognitive Science, 9, 439-444.

Kaminski, J., Riedel, J., Call, J., \& Tomasello, M. (2005). Domestic goats, Capra hircus, follow gaze direction and use social cues in an object choice task. Animal Behaviour, 69, 11-18.

Kerth, G. (2008). Causes and consequences of sociality in bats. Bioscience, 58, 737-746.

Kunz, T. H., \& Allgaier, A. L. (1994). Allomaternal care: Helper-assisted birth in the rodrigues fruit bat, Pteropus rodricensis (chiroptera: Pteropodidae). Journal of Zoology, 232, 691-700.

Kunz, T. H., \& Jones, D. P. (2000). Pteropus vampyrus. Mammalian Species, 642, 1-6. 
Luft, S., Curio, E., \& Tacud, B. (2003). The use of olfaction in the foraging behavior of the golden-mantled flying fox, Pteropus pumilus, and the greater musky fruit bat, Ptenochirus jagori (Megachiroptera: Pteropodidae). Naturwissenschaften, 90, 84-87.

Maros, K., Gácsi, M., \& Miklósi, Á. (2008). Comprehension of human pointing gestures in horses (Equus caballus). Animal Cognition, 11, 457-466.

McCracken, G. F., \& Bradbury, J. W. (1981). Social organization and kinship in the polygynous bat Pyllostomus hastatus. Behavioral Ecology and Sociobiology, 8, 11-34.

McKinley, J., \& Sambrook, T. (2000). Use of human-given cues by domestic dogs (Canis familiaris) and horses (Equus caballus). Animal Cognition, 3, 13-22.

Miklósi, Á., Kubinyi, E., Topál, J., Gácsi, M., Virányi, Z., \& Csányi, V. (2003). A simple reason for a big difference: Wolves do not look back at humans, but dogs do. Current Biology, 13, 764-766.

Miklósi, Á., Pongrácz, P., Lakatos, G., Topál, J., \& Csányi, V. (2005). A comparative study of the use of visual communicative signals in interactions between dogs (Canis familiaris) and humans and cats (Felis catus) and humans. Journal of Comparative Psychology, 119, 179-186.

Miklósi, Á., \& Soproni, K. (2006). A comparative analysis of animals' understanding of the human pointing gesture. Animal Cognition, 9, 81-93.

Müller, B., Goodman, S.M. \& Piechl, L. (2007). Cone photoreceptor diversity in the retinas of fruit bats (Megachiroptera). Brain Behavior and Evolution, 70, 90-104.

Pack, A. A., \& Herman, L. M. (2004). Bottlenosed dolphins (Tursiops truncatus) comprehend the referent of both static and dynamic human gazing and pointing in an object-choice task. Journal of Comparative Psychology, 118, 160-171.

Safi, K., \& Kerth, G. (2007). Comparative analyses suggest that information transfer promoted sociality in male bats in the temperate zone. American Naturalist, 170, 465-472.
Scheumann, M., \& Call, J. (2004). The use of experimenter-given cues by South African fur seals (Arctocephalus pusillus). Animal Cognition, 7, $224-230$.

Tomasello, M., \& Call, J. (2004). The role of humans in the cognitive development of apes revisited. Animal Cognition, 7, 213-215.

Trut, L. (1999). Early canid domestication: The farm-fox experiment. American Scientist, 87, 160-169.

Tschudin, A., Call, J., Dunbar, R. I. M., Harris, G., \& van der Elst, C. (2001). Comprehension of signs by dolphins (Tursiops truncatus). Journal of Comparative Psychology, 115, 100-105.

Udell, M. A. R., Dorey, N. R., \& Wynne, C. D. L. (2008). Wolves outperform dogs in following human social cues. Animal Behaviour, 76, 1767-1773. doi:10.1016/j.anbehav.2008.07.028

Udell, M. A. R., \& Wynne, C. D. L. (2010). Ontogeny and phylogeny: Both are essential to human-sensitive behavior in the genus Canis. Animal Behaviour, 9, e9-e14. doi:10.1016/j.anbehav.2009.11.033

Virányi, Z., Gácsi, M., Kubinyi, E., Topál, J., Belényi, B., Ujfalussy, D., \& Miklósi, Á. (2008). Comprehension of human pointing gestures in young human-reared wolves (Canis lupus) and dogs (Canis familiaris). Animal Cognition, 11, 373-387.

Von Bayern, A. M. P., \& Emery, N. J. (2009). Jackdaws respond to human attentional states and communicative cues in different contexts. Current Biology, 19, 602-606.

Wilkinson, G. S. (1984, March 8). Reciprocal food sharing in the vampire bat. Nature, 308, 181-184.

Wilkinson, G. S. (1986). Social grooming in the common vampire bat desmodus-rotundus. Animal Behaviour, 34, 1880-1889.

Wilkinson, G. S. (1992). Information transfer at evening bat colonies. Animal Behaviour, 44, 501-518.

Received February 20, 2010

Revision received January 12, 2011

Accepted January 13, 2011 